\title{
Effect of Oxygen Flow on Aerosol Delivery From a Nebulizer With a Holding Chamber
}

\author{
Rania M Sarhan, Ahmed A Elberry, Nada Sayed Abdelwahab, Hoda Rabea, \\ Mohamed Nabil Salem, and Mohamed EA Abdelrahim
}

\begin{abstract}
BACKGROUND: A new holding chamber was designed to be used with a vibrating mesh nebulizer to increase the total inhalable dose for patients. It facilitates intermittent and continuous nebulization as well as the optional supply of supplemental oxygen via a T-piece with a mouthpiece adapter. This study aimed to evaluate the effect of oxygen introduction in the new holding chamber on aerosol delivery using a vibrating mesh nebulizer. METHODS: The study was divided into 2 parts. First, the total inhalable dose of $1 \mathrm{~mL}$ of a respirable solution (nominal dose of 5,000 $\mu \mathrm{g}$ salbutamol) was determined using a breathing simulator set to provide a tidal volume of $500 \mathrm{~mL}$, a breathing frequency of 15 breaths/min, and an inspiratory:expiratory ratio of 1:1 for adults as a quiet-breathing pattern. Three experimental nebulizer setups were used: a vibrating mesh nebulizer with the holding chamber and oxygen set at $6 \mathrm{~L} / \mathrm{min}$, a vibrating mesh nebulizer with the holding chamber and no oxygen, and a vibrating mesh nebulizer with the T-piece. Aerodynamic particle size characterizations were determined using cooled Andersen cascade impaction at an inhalation flow of $15 \mathrm{~L} / \mathrm{min}$. Second, we performed an in vivo study involving 12 healthy nonsmoking subjects ( 6 female) who were $>18$ y old with an average $\mathrm{FEV}_{1}>90 \%$ of predicted. Using normal tidal breathing, subjects inhaled $1 \mathrm{~mL}$ of nebulized salbutamol $(5,000 \mu \mathrm{g})$ through the vibrating mesh nebulizer with the holding chamber with and without oxygen and through the vibrating mesh nebulizer with a T-piece. To analyze salbutamol content, urine samples were obtained 30 min after dosing as an index of lung deposition, and their urine was cumulatively collected for $24 \mathrm{~h}$ as an index of systemic absorption. RESULTS: The holding chamber significantly increased the total inhalable dose or amount of salbutamol excreted in the first $\mathbf{3 0} \mathrm{min}$, as well as the amount of salbutamol excreted over a 24-h period compared to the dose received with the vibrating mesh nebulizer with a T-piece $(P=.005, P=.034$, and $P=.02$, respectively $)$, and relatively decreased the mass median aerodynamic diameter, although the difference was not significant. However, when oxygen was introduced in the holding chamber, the total inhalable dose, or amount of salbutamol excreted in the first $30 \mathrm{~min}$, significantly decreased compared to use without oxygen $(P=.003, P=.03$ respectively). No significant difference was found between the vibrating mesh nebulizer with the holding chamber with oxygen and the vibrating mesh nebulizer with a T-piece. CONCLUSIONS: The vibrating mesh nebulizer with a holding chamber and without oxygen resulted in much better aerosol delivery compared to vibrating mesh nebulizer with a holding chamber and with oxygen delivery and to the vibrating mesh nebulizer with a T-piece. The use of oxygen with the holding chamber significantly decreased aerosol delivery and its benefit, and recommended flow should be reevaluated. Key words: nebulizer; holding chamber; T-piece; Andersen cascade impactor; vibrating mesh nebulizer. [Respir Care 2019;64(12):1508-1515. (C) 2019 Daedalus Enterprises]
\end{abstract}

\section{Introduction}

The Aerogen Ultra holding chamber (Aerogen, Galway, Ireland) was developed for use with a vibrating mesh nebulizer with a mouthpiece or mask to treat spontaneously breathing patients. Aerogen states that this holding chamber provides an aerosol reservoir and permits connection to low-flow oxygen $(1-6 \mathrm{~L} / \mathrm{min})$, and it can be used for both intermittent and continuous treatments in pediatric and adult patients. As shown in Figure 1, the device is 
composed of a valved collection chamber that connects the vibrating mesh nebulizer and a mouthpiece or face mask.

The design of the device's valved system controls flow through the aerosol chamber. Upon inhalation, the gas is drawn through the inlet valve on the base of the device, creating a flow of gas through the device. This purges the aerosol from the chamber and delivers the drug to the patient via the mouthpiece. During exhalation, the inlet valve closes, and the valve on the mouthpiece opens. This allows the patient to exhale through the port on the mouthpiece while the vibrating mesh nebulizer refills the aerosol chamber. These design features improve the efficiency of the nebulizer and decrease aerosol loss due to exhalation and condensation. ${ }^{1}$ This chamber results in better aerosol delivery compared to a T-piece with a mouthpiece adapter with the vibrating mesh nebulizers, even when connected to a jet nebulizer. ${ }^{1}$ The best delivery was found with the holding chamber designed for use with this device. ${ }^{1}$ In addition, Ari and colleagues ${ }^{2}$ reported better aerosol delivery with a vibrating mesh nebulizer connected to a holding chamber with oxygen flow of $2 \mathrm{~L} / \mathrm{min}$ compared to a jet nebulizer. However, many previous studies have reported that a vibrating mesh nebulizer without oxygen is more effective than a jet nebulizer. ${ }^{1,3-18}$ Given that the manufacturer's maximum recommended flow is $6 \mathrm{~L} / \mathrm{min}$ and the tested flow was $2 \mathrm{~L} / \mathrm{min}$, there is disagreement about the real effect of the introduction of oxygen to the holding chamber. In addition, no previous study has compared the use of a vibrating mesh nebulizer with a holding chamber with and without oxygen. We sought to evaluate the efficacy of using oxygen at the highest recommended flow $(6 \mathrm{~L} / \mathrm{min})$ with the vibrating mesh nebulizer connected to a holding chamber, and to compare this performance to that of a vibrating mesh nebulizer and holding chamber without oxygen and that of a vibrating mesh nebulizer connected to a T-piece.

\footnotetext{
Ms Sarhan and Drs Rabea and Abdelrahim are affiliated with the Clinical Pharmacy Department, Faculty of Pharmacy, Beni-suef University, Benisuef, Egypt. Dr Abdelrahim is also affiliated with the Clinical Pharmacy Department, Faculty of Pharmacy, Ahram Canadian University, Giza, Egypt. Dr Elberry is affiliated with the Clinical Pharmacology Department, Faculty of Medicine, Beni-suef University, Beni-suef, Egypt. Dr Abdelwahab is affiliated with the Analytical Chemistry Department, Faculty of Pharmacy, Beni-Suef University, Beni-Suef, Egypt. Dr Salem is affiliated with the Internal Medicine Department, Faculty of Medicine, Beni-suef University, Beni-suef, Egypt.
}

The authors have disclosed no conflicts of interest.

Correspondence: Mohamed EA Abdelrahim PhD, Department of Clinical Pharmacy, Beni-suef University, Beni-suef, Egypt. E-mail: mohamedemam9@yahoo.com.

DOI: $10.4187 /$ respcare.06639

\section{QUICK LOOK}

\section{Current knowledge}

Increasing aerosol delivery from a nebulizer can be accomplished by addition of a spacer or holding chamber. Characteristics of the holding chamber can alter delivery efficiency. The addition of oxygen has a variable impact on delivery efficiency.

\section{What this paper contributes to our knowledge}

The holding chamber increased the aerosol delivery from the mesh nebulizer compared to the T-piece alone. Concomitant use of oxygen with the holding chamber significantly decreased aerosol delivery.

\section{Methods}

\section{Amount of Aerosol Emitted (Total Inhalable Aerosol Dose)}

We used 3 formats to compare performance: the Aerogen Solo vibrating mesh nebulizer with a standard T-piece with a mouthpiece adapter, the Aerogen Solo vibrating mesh nebulizer with the Aerogen Ultra holding chamber and oxygen, and the Aerogen Solo vibrating mesh nebulizer with the Aerogen Ultra holding chamber and no oxygen. The holding chamber was attached to oxygen using the oxygen port and supply tubing connected to a flow meter, with the flow set at $6 \mathrm{~L} / \mathrm{min}$ (Fig. 2).

A breathing simulator (5600i, Michigan Instruments, Kentwood, Michigan) was used to provide a spontaneous breathing with a tidal volume of $500 \mathrm{~mL}$, a breathing frequency of 15 breaths/min, and an inhalation-exhalation ratio of $1: 1$, representing a resting breathing pattern in adults in accordance with the European Standard EN 13544-16 (CEN methodology). ${ }^{19}$ An electrostatic filter pad enclosed in a filter holder (Pari, Starnberg, Germany) was attached to the breathing simulator (inhalation filter) from one side and the nebulizer-adapter combination from the other side as shown in Figure 2. A new filter was used with each setup each time. This filter captured all of the aerosol inhaled during inspiration and thus provided a good measure of the total inhaled aerosol dose (ie, the in vitro emitted dose available for inhalation). ${ }^{13,15}$ The simulator was activated $30 \mathrm{~s}$ before aerosolized delivery of $1 \mathrm{~mL} 5,000 \mu \mathrm{g}$ salbutamol (Farcolin Respirator Solution, Pharco Pharmaceuticals, Egypt), with nebulization until the reservoir was dry.

For each combination of nebulizer and adapter, 5 determinations were made. The amounts of salbutamol deposited in the nebulizer reservoir chamber and inside the hold- 


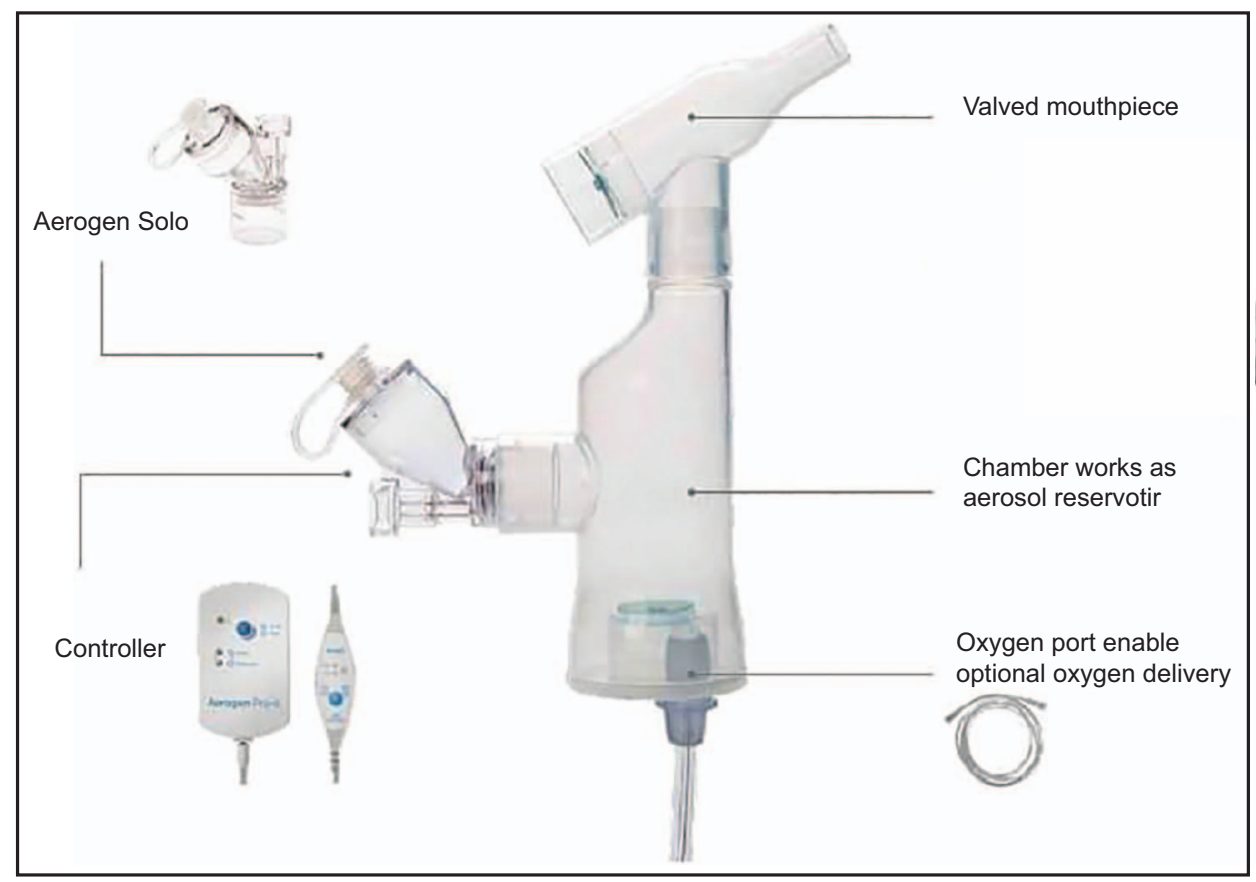

Fig. 1. Holding chamber details and connections for the Aerogen Solo nebulizer.

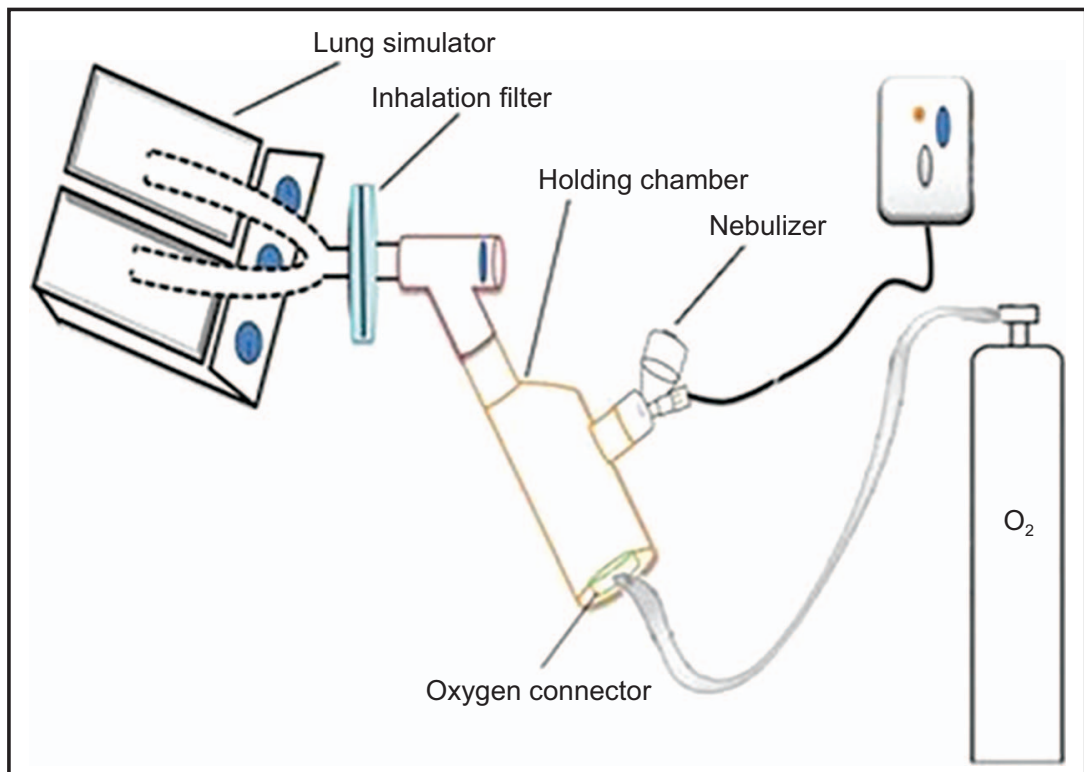

Fig. 2. Schematic diagram of the experimental setting for the determination of the amount of aerosol emitted (the total inhalable aerosol dose).

ing chamber used were recovered by washing with $20 \%$ acetonitrile, as described in our previous study. ${ }^{1}$ Salbutamol deposited on the filter was recovered by washing and sonication for $3 \mathrm{~min}$ with $20 \%$ acetonitrile. High-performance liquid chromatography with ultraviolet detection was used to identify amounts of salbutamol. No blank was run through the high-performance liquid chromatography. We used a $25 \mathrm{~mm} \times 4.6 \mathrm{~mm}$ ZORBAX Eclipse Plus C18,
ODS1 column (Agilent, Santa Clara, California), through which a mobile phase consisting of a mixture of acetonitrile and water (containing $0.1 \%$ phosphoric acid) was pumped at $1 \mathrm{~mL} / \mathrm{min}$ using an Agilent 1260 Infinity preparative pump (G1361A). The Agilent 1260 Infinity Diode array detector VL (G131SD) was set at $225 \mathrm{~nm}$ with an injection volume of $100 \mu \mathrm{L}$. Calibration solutions ranged from 4 to $100 \mu \mathrm{g} / \mathrm{mL}$ (weight/volume). The limit of de- 


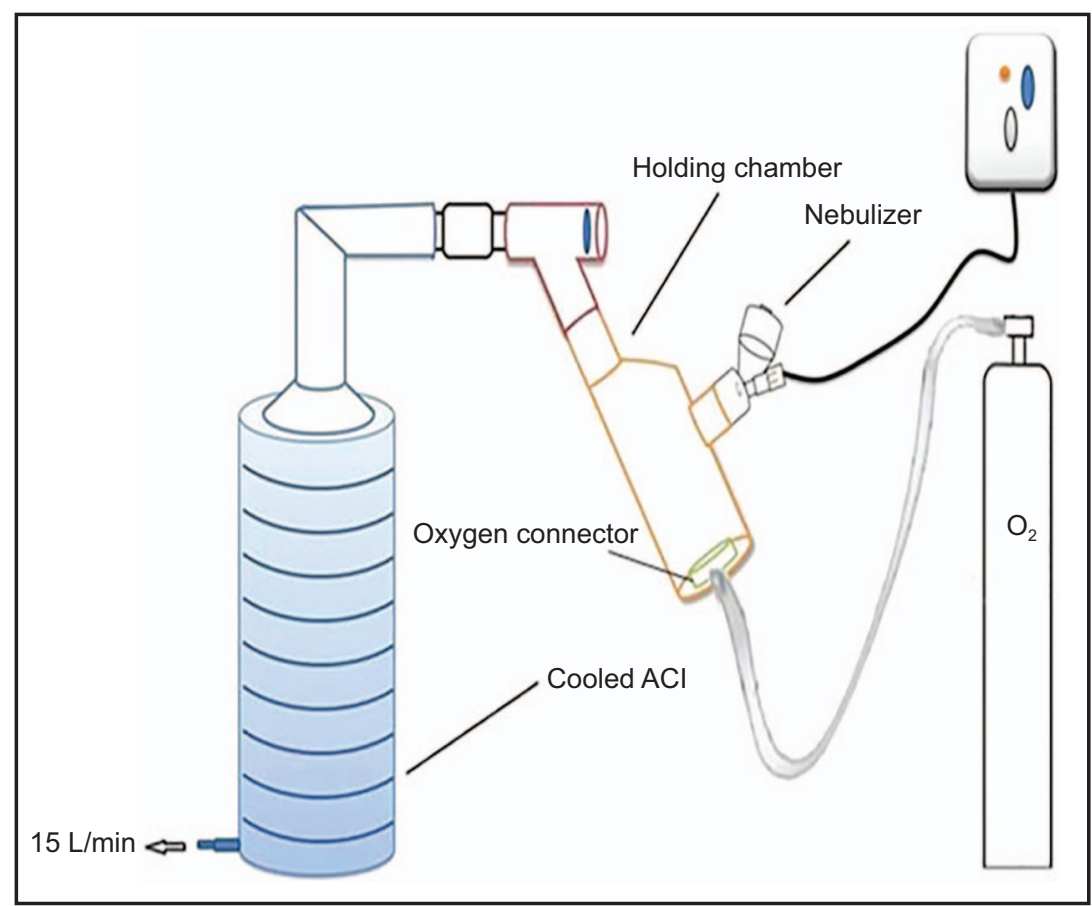

Fig. 3. Schematic diagram of the experimental setting for the determination of particle size distribution of the aerosol emitted. $\mathrm{ACl}=$ Andersen cascade impactor.

tection was $0.35 \mu \mathrm{g} / \mathrm{mL}$, and the lower limit of quantification was $2.55 \mu \mathrm{g} / \mathrm{mL}$.

\section{Particle Size Distribution of the Aerosol Emitted}

A cooled Andersen cascade impactor (Copley Scientific, Nottingham, United Kingdom) was used to determine the size distribution of particle droplets of the aerosolized medication. The cascade impactor, with its plates in situ, was placed in a refrigerator at $5^{\circ} \mathrm{C}$ for $60 \mathrm{~min}$ before use. ${ }^{17}$ Immediately after removing the cascade impactor from the refrigerator, the inspiratory flow was adjusted to $15 \mathrm{~L} / \mathrm{min}$, and the induction port of the cascade impactor was connected directly to the mouthpiece of the nebulizer-adapter combination tested as shown in Figure 3 . The vacuum flow through the cascade impactor was provided by a vacuum pump (Brook Crompton, United Kingdom). The flow was measured using an electronic digital flow meter (MKS Instruments, Andover, Massachusetts). The respirable salbutamol solution was nebulized until the fill cup was dry. For each nebulizer adapter combination, 3 determinations were made.

Salbutamol deposited on each plate of the cascade impactor, in nebulizer reservoir chamber, and in the adaptors was recovered by rinsing with $20 \%$ acetonitrile. Similarly, the mass entrained on the filter was recovered with sonication and rinsing. High-performance liquid chromatography was used as previously described.
The fine particle dose, fine-particle fraction, and the mass median aerodynamic diameter (MMAD) were determined using Copley Inhaler Testing Data Analysis Software (CITDAS, Copley Scientific) impactor data.

\section{In Vivo Study}

Hindle and Chrystyn ${ }^{20}$ developed a urinary pharmacokinetic method to determine relative lung and systemic bioavailability of salbutamol following inhalation. This method used the amount of drug excreted in the first $30 \mathrm{~min}$ as an index of lung deposition and the amount of drug excreted over a 24-h period after inhalation as an index of systemic absorption. ${ }^{20}$ This noninvasive pharmacokinetic method has been used to detect lung deposition of aerosolized drug in healthy volunteers, ${ }^{21}$ in subjects admitted with an exacerbation of either asthma or COPD, 22 and in ventilated subjects. $., 6,12,23,24$ This method has also been used to detect aerosol drug deposition in subjects receiving low-flow oxygen via a nasal cannula. ${ }^{25} \mathrm{We}$ used similar methodology to compare lung deposition and systemic absorption.

This study was conducted following the amended Declaration of Helsinki. Local institutional review boards and independent ethics committees approved the protocol, and written informed consent was obtained from all subjects. Twelve healthy nonsmoking subjects (6 female) participated in the study. The subjects had a mean $\pm \mathrm{SD}$ age, weight, and height of $33.3 \pm 5.6 \mathrm{y}, 82.7 \pm 7.4 \mathrm{~kg}$, and 


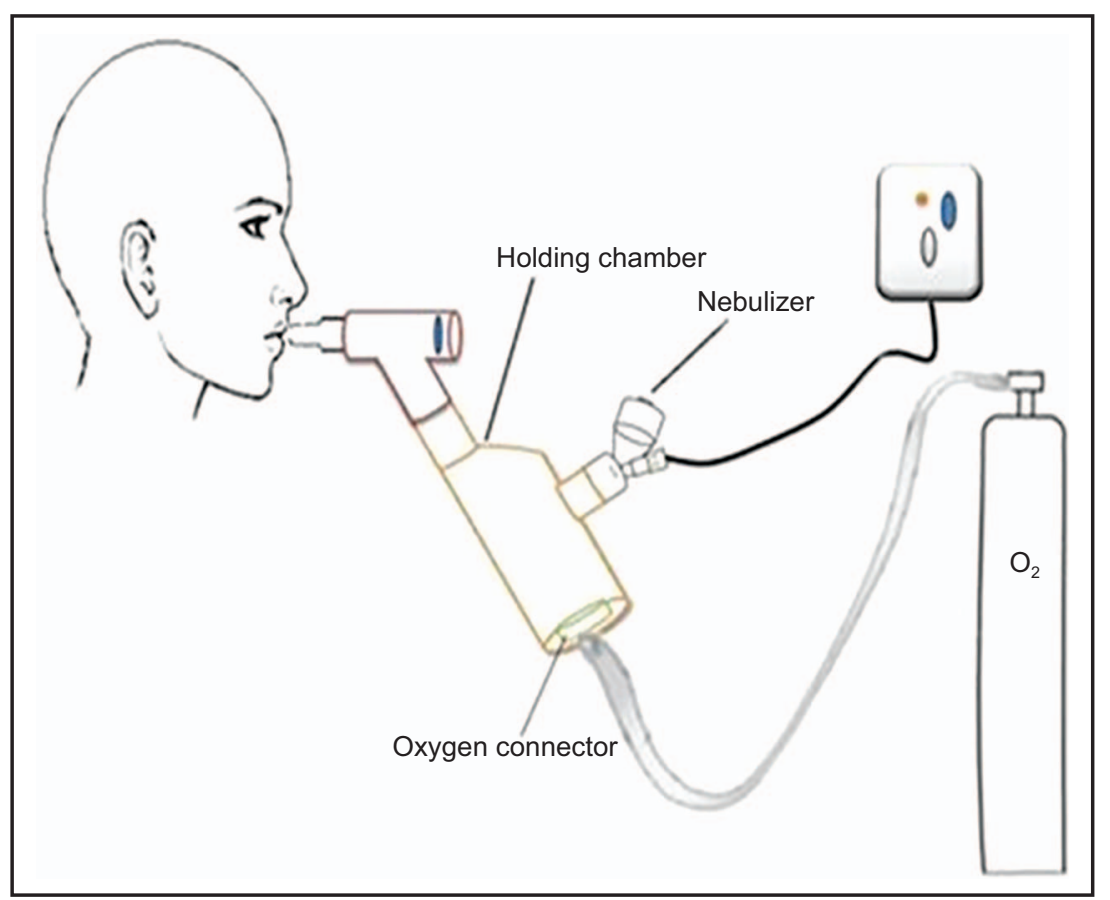

Fig. 4. Schematic diagram of in vivo methodology indicating the position of the nebulizer to volunteer with the oxygen source.

$168.0 \pm 4.9 \mathrm{~cm}$, respectively. The $\mathrm{FEV}_{1}$ of all subjects was $>90 \%$ of predicted with a mean \pm SD of $95.4 \% \pm 4.0 \%$ of predicted. Subjects were first trained on the breathing technique with the nebulizer. Subjects were trained to place the mouthpiece between their lips and breathe in and out gently through their mouth. Subjects were randomly assigned to receive aerosolized medication from each of the 3 study setups, as previously described and shown in Figure 4, with a 7-d washout period between dosing. The dose was loaded in the vibrating mesh nebulizer for the subject before use according to the patient information leaflet.

Subjects were asked to provide a urine sample $30 \mathrm{~min}$ after the start of the dosing as an index of salbutamol delivery to the lungs. ${ }^{20}$ Subjects also were asked to collect all their urine over the next $24 \mathrm{~h}$ in one container as an index of systemic absorption of salbutamol after inhalation. ${ }^{20}$ We measured the volumes of excreted drug in the first $30 \mathrm{~min}$ and over a 24-h period, and we then used high-performance liquid chromatography to assay the samples. Bambuterol hydrochloride was added as the internal standard to the collected urine samples. Salbutamol and bambuterol were extracted from the urine sample using solid-phase extraction. ${ }^{6}$ The eluent was then injected into the high-performance liquid chromatography system.

\section{Statistical Analysis}

All data are expressed as mean (SD). One-way analysis of variance with the application of least significant differ- ence correction was used to compare the 3 nebulizer adapter combinations. The urine samples were compared using the Kruskal-Wallis analysis of variance followed by the MannWhitney test for pairwise comparison using SPSS V15.0 (SPSS, Chicago, Illinois).

\section{Results}

Table 1 provides a summary of the outcome of the delivered dose. The vibrating mesh nebulizer and holding chamber without oxygen significantly increased the total mean \pm SD inhaled dose deposited on the inhalation filter $(2,197.7 \pm 470.7 \mu \mathrm{g})$ compared to the vibrating mesh nebulizer and holding chamber with oxygen $(1,081.5 \pm 333.9 \mu \mathrm{g}, P=.003)$ or the vibrating mesh nebulizer with T-piece $(1,351.6 \pm 198.8 \mu \mathrm{g}, P=.005)$. No significant difference was found in the total inhaled dose between the vibrating mesh nebulizer with a T-piece and the vibrating mesh nebulizer with the holding chamber and oxygen, with relatively higher results from the vibrating mesh nebulizer with a T-piece. The amount deposited inside the holding chamber without oxygen $(820.7 \pm 114.9 \mu \mathrm{g})$ was significantly lower that than in the vibrating mesh nebulizer with a holding chamber and oxygen $(454.7 \pm 135.8 \mu \mathrm{g}, P=.004)$ or the T-piece $(603.2 \pm 76.5 \mu \mathrm{g}$, $P=.034)$. The amount deposited inside the holding chamber with oxygen was relatively lower than in the T-piece, although the difference was not significant. 
Table 1. Outcomes Using a Vibrating Mesh Nebulizer With a Holding Chamber With and Without Oxygen and With a T-Piece Alone

\begin{tabular}{lccc}
\hline \hline & \multicolumn{3}{c}{ Vibrating Mesh Nebulizer } \\
\cline { 2 - 4 } & Without Oxygen & With Oxygen & With a T-Piece \\
\hline Total inhaled dose, $\mu \mathrm{g}$ & $2,197.7 \pm 470.7$ & $1,081.5 \pm 333.9$ & $1,351.6 \pm 198.8$ \\
Nebulizer residual, $\mu \mathrm{g}$ & $186.8 \pm 74.0$ & $176.2 \pm 33.69$ & $261.1 \pm 98.0$ \\
Amount deposited inside the holding chamber, $\mu \mathrm{g}$ & $820.7 \pm 114.9$ & $454.7 \pm 135.8$ & $603.2 \pm 76.5$ \\
Fine-particle fraction, \% & $64.5 \pm 3.6$ & $64.3 \pm 3.6$ & $67.9 \pm 1.7$ \\
Mass median aerodynamic diameter, $\mu \mathrm{m}$ & $2.3 \pm 1.2$ & $2.4 \pm 0.1$ & $2.9 \pm 0.5$
\end{tabular}

Data are presented as mean $\pm \mathrm{SD}$. Trials involved a nominal dose of $1 \mathrm{~mL}$ respirable solution of salbutamol $(5,000 \mu \mathrm{g}$ nominal dose $)$ delivered using a vibrating mesh nebulizer with a holding chamber with and without oxygen at $6 \mathrm{~L} / \mathrm{min}$ or using a T-piece alone

Table 2. Salbutamol Excretion After Inhalation Using a Vibrating Mesh Nebulizer With a Holding Chamber With and Without Oxygen and With a T-Piece Alone

\begin{tabular}{lccc}
\hline \hline & & Vibrating Mesh Nebulizer \\
\cline { 2 - 3 } & Without Oxygen & With Oxygen & With a T-Piece \\
\hline $\begin{array}{l}\text { Salbutamol excreted in the first } 30 \mathrm{~min} \\
\quad \text { after start of inhalation, } \mu \mathrm{g}\end{array}$ & $110.1 \pm 82.7$ & $80.4 \pm 30.9$ \\
$\begin{array}{l}\text { Salbutamol excreted over a 24-h period, } \mu \mathrm{g} \\
\begin{array}{l}\text { Data are presented as mean } \pm \text { SD. Trials involved a nominal dose of } 1 \mathrm{~mL} \text { respirable solution of salbutamol (5,000 } \mu \mathrm{g} \text { nominal dose) delivered using a vibrating mesh nebulizer with a holding } \\
\text { chamber with and without oxygen at } 6 \mathrm{~L} / \mathrm{min} \text { or using a T-piece alone. }\end{array}\end{array}$ \\
\hline
\end{tabular}

The aerodynamic droplet size distributions from the 3 nebulizer-adapter combinations are shown in Table 1. No significant difference was found in the fine-particle fraction and the MMAD.

The mean $\pm \mathrm{SD}$ amounts of salbutamol excreted after inhalation from the 3 nebulizer-adapter combinations are presented in Table 2. The amount of salbutamol excreted in the first 30 min after the start of the inhalation was significantly higher from the vibrating mesh nebulizer with the holding chamber and no oxygen $(110.1 \pm 82.7 \mu \mathrm{g})$ than from the vibrating mesh nebulizer with the holding chamber and with oxygen $(80.4 \pm 30.9 \mu \mathrm{g}, P=.03)$ or from the vibrating mesh nebulizer with a T-piece (84.8 $\pm 45.3 \mu \mathrm{g}, P=.034$ ). The amount of salbutamol excreted over a $24-\mathrm{h}$ period after inhalation from the vibrating mesh nebulizer with the holding chamber and no oxygen $(906.1 \pm 572.6 \mu \mathrm{g})$ was significantly higher than that from the vibrating mesh nebulizer with a T-piece (517.5 $\pm 332.6 \mu \mathrm{g}, P=.02$ ).

No significant difference was found in the amount of salbutamol excreted in the first $30 \mathrm{~min}$ and over a 24-h period between the vibrating mesh nebulizer with a T-piece and the vibrating mesh nebulizer with the holding chamber and oxygen.

\section{Discussion}

The holding chamber was previously reported to deliver a significantly higher amount of aerosol compared to a
T-piece without the introduction of oxygen. ${ }^{1}$ The results of our study indicate a significantly increased total inhaled dose $(P=.005)$, most likely resulting from a reduction in ambient aerosol loss with the use of the holding chamber. Potential condensation on the wall of the T-piece, influenced by inertial impaction and gravity, should have been minimized because the holding chamber and the vibrating mesh nebulizer were placed side by side to reduce the effects of gravitational sedimentation (Fig. 2), whereas the holding chamber decreased impaction. ${ }^{1}$

The use of the holding chamber with the vibrating mesh nebulizer did not have much effect on the aerodynamic characterization; the only noted effect was a relatively insignificant decrease in the MMAD. This was due to the nature of the nebulized aerosol, which does not contain a propellant as in a metered-dose inhaler, and thus allows evaporation to take place when using a holding chamber or spacers. ${ }^{9,26}$ However, the main effect of the holding chamber is not to decrease the particle size of the aerosolized medication but to prevent ambient loss of the aerosol. ${ }^{27}$

The urinary data from the in vivo study showed similar results; the vibrating mesh nebulizer with the holding chamber significantly increased the amount of salbutamol excreted over a 24 -h period by 1.8 -fold $(P=.02)$ and the amount of drug excreted in the first $30 \mathrm{~min}$ by 1.3 -fold $(P=.034)$ compared to vibrating mesh nebulizer with a T-piece. These results suggest that the total inhaled dose (1.6-fold, $P=.005$ ) is more closely related to the amount 
delivered to the subject, not the fraction deposited into the lung.

Thus, the holding chamber delivered more medication to the subject in the first $30 \mathrm{~min}$ and over a subsequent 24-h period. This increased delivery would be accompanied by a reduction of the aerosol loss, which may also be inhaled by the health care provider in the vicinity of the patient. ${ }^{7,28}$ Hence, we recommend the use of the holding chamber with the vibrating mesh nebulizer for better aerosol delivery.

The introduction of oxygen at the highest recommended flow $(6 \mathrm{~L} / \mathrm{min})$ dramatically decreased aerosol delivery, as indicated by the amount of salbutomol excreted in the first $30 \mathrm{~min}$ and over a $24-\mathrm{h}$ period, to the extent that there were no significant differences between the vibrating mesh nebulizer with the holding chamber with oxygen and the vibrating mesh nebulizer with the holding chamber and a T-piece. In other words, the use of oxygen at the highest recommended flow eliminated the benefit of the holding chamber.

Initially, these findings appear to counter those of Ari et al, ${ }^{2}$ who reported better aerosol delivery with a vibrating mesh nebulizer connected to a holding chamber with oxygen flow at $2 \mathrm{~L} / \mathrm{min}$ compared to the jet nebulizer. As previously noted, however, this comparison was between a vibrating mesh nebulizer and a jet nebulizer, whereas our study compared different interfaces and oxygen flows with variations of a vibrating mesh nebulizer. In addition, the vibrating mesh nebulizer has been reported to provide greater aerosol delivery than the jet nebulizer due to the vibrating mesh nebulizer's greater efficiency, regardless of setup. ${ }^{1,3-18}$

Higher oxygen flows may flush the aerosol out of the chamber, diluting the delivered dose. With no oxygen flow or at lower flows, the aerosol collects in the holding chamber and is available to the patient on inspiration. The volume of the holding chamber was $130 \mathrm{~mL}$, and a flow of $6 \mathrm{~L} / \mathrm{min}$ was equal to $100 \mathrm{~mL} / \mathrm{s}$. In addition, the breathing simulator was set to a tidal volume of $500 \mathrm{~mL}$, a breathing frequency of 15 breaths/min, and an inhalation-exhalation ratio of $1: 1$. Thus, with an expiration of $2 \mathrm{~s}$, which is equivalent to $200 \mathrm{~mL}$ of oxygen, most of the aerosol in the holding chamber was flushed out before inhalation. Therefore, the higher the oxygen flow, the lower the amount of aerosol collected in the holding chamber between breaths. In light of this finding, it would be interesting to compare inhaled dose across different tidal volumes, inspiratory patterns, and flows to determine how the inhaled dose correlates to the calculated dose with the holding chamber.

Our study has several limitations. We only used the highest oxygen flow recommended by the manufacturer (ie, $6 \mathrm{~L} / \mathrm{min}$ ). The coefficient of variation for urinary salbutamol was reported as approximately double that for plasma salbutamol, which would account for some of the nonsignificant difference. ${ }^{29}$ Finally, the urinary excretion method provides a 30-min index of salbutamol delivery to the lungs rather than of regional lung deposition.

\section{Conclusions}

The holding chamber had little effect on MMAD and fine-particle fraction. Using the holding chamber with the vibrating mesh nebulizer significantly increased aerosol delivery compared to the setup with a T-piece. The vibrating mesh nebulizer with a holding chamber had the highest total inhaled dose, as demonstrated by the amount of salbutamol excreted in the first $30 \mathrm{~min}$ as an index of lung deposition and by the amount of salbutamol excreted over a 24-h period as an index of systemic absorption. Introducing oxygen at the maximum recommended flow $(6 \mathrm{~L} / \mathrm{min})$ within the holding chamber resulted in a significant decrease in aerosol delivery. Different tidal volumes, inspiratory patterns, and oxygen flows should be studied further due to the potential to decrease aerosol delivery.

\section{ACKNOWLEDGMENTS}

Dr Yousef Hady and Dr Haitham Saeed provided help in English editing.

\section{REFERENCES}

1. Sarhan RM, Elberry AA, Abdelwahab NS, Rabea H, Salem MN, Abdelrahim ME. Effect of a nebulizer holding chamber on aerosol delivery. Respir Care 2018:63(9):1125-1131.

2. Ari A, Dornelas de Andrade A, Sheard M, AlHamad B, Fink JB. Performance comparisons of jet and mesh nebulizers using different interfaces in simulated spontaneously breathing adults and children. J Aerosol Med Pulm Drug Deliv 2015;28(4):281-289.

3. Saeed H, Ali AMA, Elberry AA, Eldin AS, Rabea H, Abdelrahim ME. Modeling and optimization of nebulizers' performance in noninvasive ventilation using different fill volumes: Comparative study between the vibrating mesh and jet nebulizers. Pulm Pharmacol Ther 2018;50:62-71.

4. Saeed H, Mohsen M, Salah Eldin A, Elberry AA, Abdelwahab NS, Hussein RRS, et al. Effects of fill volume and humidification on aerosol delivery during single limb non-invasive ventilation. Respir Care 2018:63(11):1370-1378.

5. Moustafa IOF, Ali MRA, Al Hallag M, Rabea H, Fink JB, Dailey P, et al. Lung deposition and systemic bioavailability of different aerosol devices with and without humidification in mechanically ventilated patients. Heart Lung 2017;46(6):464-467.

6. ElHansy MHE, Boules ME, El-Essawy AFM, Al-Kholy MB, Abdelrahman MM, Said ASA, et al. Inhaled salbutamol dose delivered by jet nebulizer, vibrating mesh nebulizer and metered dose inhaler with spacer during invasive mechanical ventilation. Pulm Pharmacol Ther 2017;45:159-163.

7. Saeed H, Mohsen M, Fink JB, Dailey P, Salah Eldin A, Abdelrahman MM, et al. Fill volume, humidification and heat effects on aerosol delivery and fugitive emissions during noninvasive ventilation. J Drug Deliv Sci Technol 2017;39:372-378.

8. Moustafa IOF, ElHansy MHE, Al Hallag M, Fink JB, Dailey P, Rabea $\mathrm{H}$, et al. Clinical outcome associated with the use of different inhalation method with and without humidification in asthmatic mechanically ventilated patients. Pulm Pharmacol Ther 2017;45:40-46. 


\section{Oxygen Flow With a Holding Chamber}

9. Madney YM, Fathy M, Elberry AA, Rabea H, Abdelrahim MEA. Nebulizers and spacers for aerosol delivery through an adult nasal cannula at a low oxygen flow rate: an in-vitro study. J Drug Deliv Sci Technol 2017;39:260-265.

10. Mohsen M, Elberry AE, Salah Eldin A, Hussein RR, Abdelrahim EM. Effects of heat and humidification on aerosol delivery during auto-CPAP noninvasive ventilation. Arch Pulm Respir Care 2017; 3(1):11-15.

11. Saeed H, Elberry AA, Eldin AS, Rabea H, Abdelrahim MEA. Effect of nebulizer designs on aerosol delivery during non-invasive mechanical ventilation: a modeling study of in vitro data. Pulm Ther 2017;3(1):233-241.

12. Hassan A, Salah Eldin R, Abdelrahman MM, Abdelrahim ME. In-vitro/ in-vivo comparison of inhaled salbutamol dose delivered by jet nebulizer, vibrating mesh nebulizer and metered dose inhaler with spacer during non-invasive ventilation. Exp Lung Res 2017;43(1):19-28.

13. Abdelrahim ME, Plant P, Chrystyn H. In-vitro characterization of the nebulized dose during non-invasive ventilation. J Pharm Pharmacol 2010;62(8):966-972.

14. ElHansy MHE, Boules ME, Farid H, Chrystyn H, El-Maraghi SK, Al-Kholy MB, et al. In vitro aerodynamic characteristics of aerosol delivered from different inhalation methods in mechanical ventilation. Pharm Dev Technol 2017;22(6):844-849.

15. Hassan A, Rabea H, Hussein RRS, Salah Eldin R, Abdelrahman MM, Said ASA, et al. In-vitro characterization of the aerosolized dose during non-invasive automatic continuous positive airway pressure ventilation. Pulm Ther 2016;2(1):115-126.

16. Abdelrahim M, Plant $\mathrm{P}$, Chrystyn $\mathrm{H}$. The relative lung and systemic bioavailability of terbutaline following nebulization in non-invasively ventilated patients. Int J Pharm 2011;420(2):313-318

17. Abdelrahim ME. Aerodynamic characteristics of nebulized terbutaline sulfate using the Andersen Cascade Impactor compared to the Next Generation Impactor. Pharm Dev Technol 2011;16(2):137-145.

18. Abdelrahim ME, Chrystyn H. Aerodynamic characteristics of nebulized terbutaline sulphate using the Next Generation Impactor (NGI) and CEN method. J Aerosol Med Pulm Drug Deliv 2009;22(1):19-28.

19. Comité-Européen-Normalisation. Respiratory therapy equipmentpart 1: nebulizing system and their components. prEN 13544-1. Brussels: CEN, 2001:33-38.
20. Hindle M, Chrystyn H. Determination of the relative bioavailability of salbutamol to the lung following inhalation. Br J Clin Pharmacol 1992;34(4):311-315.

21. Silkstone V, Dennis J, Pieron C, Chrystyn H. An investigation of in vitro/in vivo correlations for salbutamol nebulized by eight systems. J Aerosol Med 2002;15(3):251-259.

22. Mazhar SH, Ismail NE, Newton DA, Chrystyn H. Relative lung deposition of salbutamol following inhalation from a spacer and a Sidestream jet nebulizer following an acute exacerbation. Br J Clin Pharmacol 2008;65(3):334-337.

23. Rabea H, Ali AMA, Salah Eldin R, Abdelrahman MM, Said ASA, Abdelrahim ME. Modelling of in-vitro and in-vivo performance of aerosol emitted from different vibrating mesh nebulisers in non-invasive ventilation circuit. Eur J Pharm Sci 2017;97 182-191.

24. Hussein RRS, M A Ali A, Salem HF, Abdelrahman MM, Said ASA, Abdelrahim MEA. In vitro/in vivo correlation and modeling of emitted dose and lung deposition of inhaled salbutamol from metered dose inhalers with different types of spacers in noninvasively ventilated patients. Pharm Dev Technol 2017;22(7):871-880.

25. Madney YM, Fathy M, Elberry AA, Rabea H, Abdelrahim ME. Aerosol delivery through adult nasal-cannula via HFNC circuit at a low-oxygen flow. Respir Care, in press

26. Harb HS, Elberry AA, Rabea H, Fathy M, Abdelrahim MEA. Is Combihaler usable for aerosol delivery in single limb non-invasive mechanical ventilation? J Drug Deliv Sci Technol 2017;40: 28-34.

27. Dugernier J, Hesse M, Vanbever R, Depoortere V, Roeseler J, Michotte J-B, et al. SPECT-CT comparison of lung deposition using a system combining a vibrating-mesh nebulizer with a valved holding chamber and a conventional jet nebulizer: a randomized cross-over study. Pharm Res 2017;34(2):290-300.

28. Ari A, Fink JB, Pilbeam SP. Secondhand aerosol exposure during mechanical ventilation with and without expiratory filters: an invitro study. Indian J Respir Care 2016;5(1):1-6.

29. Clark D, Lipworth B. Effect of multiple actuations, delayed inhalation and antistatic treatment on the lung bioavailability of salbutamol via a spacer device. Thorax 1996;51(10):981-984. 